\title{
Variable stars in the globular cluster M 28 (NGC 6626) $)^{\star \star \star \star}$ (Research Note)
}

\author{
G. Prieto ${ }^{1,2}$, M. Catelan ${ }^{1,3}$, R. Contreras Ramos ${ }^{1,4}$, B. J. Pritzl ${ }^{5}$, H. A. Smith ${ }^{6}$, and J. Alonso-García ${ }^{1,3}$ \\ 1 Pontificia Universidad Católica de Chile, Facultad de Física, Departamento de Astronomía y Astrofísica, \\ Av. Vicuña Mackenna 4860, 782-0436 Macul, Santiago, Chile \\ 2 Las Campanas Observatory, Carnegie Institution of Washington, Colina El Pino, Casilla 601, La Serena, Chile \\ 3 The Milky Way Millennium Nucleus, Av. Vicuña Mackenna 4860, 782-0436 Macul, Santiago, Chile \\ 4 Dipartimento di Astronomia, Università di Bologna, via Ranzani 1, 40127 Bologna, Italy \\ 5 Department of Physics \& Astronomy, University of Wisconsin-Oshkosh, Oshkosh, WI 54901, USA \\ 6 Dept. of Physics and Astronomy, Michigan State University, East Lansing, MI 48824, USA
}

Received 25 November 2011 / Accepted 20 June 2012

\section{ABSTRACT}

\begin{abstract}
Context. We present a new search for variable stars in the Galactic globular cluster M 28 (NGC 6626).
Aims. The search is based on a series of BVI images obtained with the SMARTS Consortium's $1.3 \mathrm{~m}$ telescope at Cerro Tololo Inter-American Observatory, Chile.

Methods. The search was carried out using the ISIS v2.2 image subtraction package.

Results. We find a total of 25 variable stars in the field of the cluster, nine being new discoveries. Of the newly found variables, one is an ab-type RR Lyrae star, six are c-type RR Lyrae, and two are long-period/semi-regular variables. V22, previously classified as a type II Cepheid, appears as a bona-fide RRc in our data. In turn, V20, previously classified as an ab-type RR Lyrae, could not be properly phased with any reasonable period.

Conclusions. The properties of the ab-type RR Lyrae stars in M 28 appear most consistent with an Oosterhoff-intermediate classification, which is unusual for bona-fide Galactic globulars clusters. However, the cluster's c-type variables do not clearly support such an Oosterhoff type, and a hybrid Oosterhoff I/II system is accordingly another possibility, thus raising the intriguing possibility of multiple populations being present in M 28. Coordinates, periods, and light curves in differential fluxes are provided for all the detected variables.
\end{abstract}

Key words. globular clusters: individual: M 28 (NGC 6626) - stars: evolution - stars: variables: RR Lyrae

\section{Introduction}

M 28 (NGC 6266) is a moderately reddened $[E(B-V)=$ $0.40 \mathrm{mag}]$ and bright $\left(M_{V}=-8.16 \mathrm{mag}\right)$ globular cluster $(\mathrm{GC})$ located at a low Galactic latitude $(b=-5.58)^{1}$. Though relatively close, at a distance from the Sun of only $5.5 \mathrm{kpc}$, it remains a relatively ill-studied cluster, likely due to the unfavorable (and highly variable) foreground reddening. Indeed, variability studies of the cluster have so far been mostly restricted to photographic data (Wehlau \& Butterworth 1990; Wehlau \& Clement 1990), and modern color-magnitude diagrams (CMDs) have only recently been presented in the literature (Davidge et al. 1996; Rosenberg et al. 2000; Testa et al. 2001; Alonso-García et al. 2012).

Still, M 28 appears as a particularly interesting object for at least three reasons: first, it has been found to have a disk-like orbit (Rees \& Cudworth 1991; Cudworth \& Hanson 1993), thus making it one of the most metal-poor members of the so-called "thick disk" family of GCs (see, e.g., Armandroff \& Zinn 1988). Second, among clusters of similar metallicity $([\mathrm{Fe} / \mathrm{H}] \simeq-1.32)$,

* Based on observations obtained with the SMARTS Consortium $1.3 \mathrm{~m}$ telescope at the Cerro Tololo Inter-American Observatory, Chile.

$\star \star$ Figures 2-5 are available in electronic form at

http://www . aanda.org

1 Unless otherwise noted, all cluster parameters in this paper are from Harris (1996, Dec. 2010 update).
M 28 stands out as having a horizontal branch (HB) morphology strongly skewed towards the blue (see also Alcaino 1981), thus making it a "second parameter" cluster. The latter, if interpreted in terms of age, would accordingly indicate that the metal-poor tail of the thick disk is at least as old as the oldest components of the Galactic halo. Third, the cluster has been classified into an Oosterhoff $(1939,1944)$ I (OoI) type, which is consistent with the relatively high metallicity of the cluster but in conflict with its HB type: with rare exceptions, blue HB clusters are commonly associated with type OoII (Contreras et al. 2005, 2010).

According to the Clement et al. (2001) catalog ${ }^{2}$, there are at present 24 variable stars known in the field of the cluster (in addition to a millisecond pulsar; Lyne et al. 1987). Of these, V1-V16 were discovered by Sawyer (1949), whereas V17-V24 were first discovered/reported on by Wehlau \& Sawyer Hogg (1982, 1984). Updated ephemerides for these stars were provided in Wehlau \& Butterworth (1990) and Wehlau \& Clement (1990), which remain the most recent papers to deal with the (photographic) light curves of M 28 variable stars in a systematic way. Rees \& Cudworth (1991) reported, again based on photographic plates, on three additional candidate variables in the direction of the cluster, which however have not yet been systematically studied, or even incorporated into the electronic version of the Clement et al. catalog.

${ }^{2}$ http://www. astro.utoronto.ca/ cclement/read.html 
There are strong reasons to believe that the current variable star tally for M 28 is incomplete. First, while M 28 is a very concentrated cluster, the photographic material which has been used in all previous variability studies does not allow the cluster core to be reliably resolved. Second, modern image-subtraction techniques (e.g., Alard \& Lupton 1998; Alard 2000; Bramich 2008), when applied to modern CCD images, have recently been providing rich harvests of variable stars towards the centers of even the previously best studied GCs (see, e.g., Contreras et al. 2010; Kuehn et al. 2011, for recent examples). Accordingly, the main purpose of this paper is to provide the first CCD-based timeseries photometry for the central regions of $\mathrm{M} 28$, in order to search for additional variables that may have gone unnoticed in previous studies and to provide updated periods and light curves for previously studied variables.

We begin in Sect. 2 by describing out dataset and reduction techniques. In Sect. 3, we describe our variability results. A summary of our results is finally provided in Sect. 4.

\section{Observations and data reduction}

Our variability search is based on a total of 39 images in each of the Johnson-Cousins BVI filters, obtained in service mode with the SMARTS $1.3 \mathrm{~m}$ telescope at CTIO. The exposure times were 70,25 , and $15 \mathrm{~s}$ in $B, V$, and $I$, respectively. The images cover the period between June 2 and July 24, 2004, with 6 to 10 images obtained every 3 to 5 days.

The images were reduced in the standard way, using IRAF tasks ${ }^{3}$. A search for stellar variability was performed using ISIS v2.2 (Alard \& Lupton 1998; Alard 2000), but modifying the search module so as to include candidates from all the available filters. For the variable star candidates detected, periods of variability were obtained using phase dispersion minimization (PDM; Stellingwerf 1978).

\section{Variable stars}

Based on the variability search conducted with ISIS, we were able to find 25 variable stars with good photometry to reveal clean light curves. A finding chart with the new discoveries is given in Fig. 1. (For clarity, previously known variables are not shown in this chart. Finding charts indicating the positions of those variables, including those that fall outside our field of view, are provided by Wehlau \& Sawyer Hogg 1984.) Light curves for the individual variable stars (in relative flux units) are provided in Figs. 2 to 5. The detected variables include 6 ab-type RR Lyrae, only one of which is a new discovery. In addition, 9 c-type RR Lyrae were found, 6 of which being new discoveries, of which one is a possible field interloper. The previously reported RR Lyrae stars are confirmed in our data, as are the 4 type II Cepheids. However, two of these candidate Cepheids could not be cleanly classified on the basis of our data. In particular, for V21 we are not able to phase the data properly, which might suggest that the star is a long-period variable (LPV). This is surprising, since Wehlau \& Sawyer Hogg (1984) present a nicely phased light curve for this star, using a period of $29.93 \mathrm{~d}$. Unfortunately, the star appears saturated in the Alonso-García et al. (2012) study; however, its position in our own preliminary

\footnotetext{
3 IRAF is distributed by the National Optical Astronomy Observatories, which are operated by the Association of Universities for Research in Astronomy, Inc., under cooperative agreement with the National Science Foundation.
}

CMD (not shown) is not inconsistent with an LPV classification. For V22, instead of the favored period of $0.99538 \mathrm{~d}$ in the 2009 version of the Clement et al. (2001) catalog, which also comes from Wehlau \& Sawyer Hogg (1984), our data favor a much shorter period, of $0.323 \mathrm{~d}$, and so this star may be better classified as a c-type RR Lyrae. The position of the star in the Alonso-García et al. CMDs is fully consistent with a c-type classification. In Wehlau \& Sawyer Hogg (1982) that reported the discovery of V22, a period of $0.498828 \mathrm{~d}$ was originally given, whereas in Wehlau \& Sawyer Hogg (1984) a period of $0.498 \mathrm{~d}$ was given as an alternative, with a note also emphasizing that the star is blended in their images, which is supported by the large amount of scatter in their derived light curve. V21 and V22 are both fairly close to the center for such early photographic work.

The case of V20 is particularly intriguing. While previously a period of $0.49774 \mathrm{~d}$ was reported (Wehlau \& Sawyer Hogg 1984), and the star classified as an ab-type RR Lyrae, we could not phase our data properly with this period. A period around $0.4948 \mathrm{~d}$ provides a better fit to our data, though with significant scatter. While this is consistent with Wehlau \& Sawyer Hogg's comment that the star is blended, its mean magnitude in our preliminary photometry is not noticeably brighter than the magnitudes of other RR Lyrae stars in the cluster, and neither is it much redder than many of the other RR Lyrae. The light curve shape supports an ab-type classification, even though a cleaner light curve for a definitive assessment would be desirable. The star's position in the Alonso-García et al. (2012) CMDs is also fully consistent with an RRab classification. Unfortunately, our dataset is insufficient for an assessment of the incidence of the Blazhko (1907) effect among our studied stars.

As to the remaining stars, namely V2, V3, V7, and V10, we confirm the results of previous studies, in that the stars appear to vary on long timescales. For V2 and V3, we provide periods for the first time. V7 had a previously reported period of $320 \mathrm{~d}$, which is much too long a timescale for our dataset to provide meaningful information regarding periodicity. In any case, its position in the Alonso-García et al. (2012) CMDs clearly confirm that it is a fairly metal-rich red giant star in the background. Finally, for V10 we are again unable to phase the light curve with a significant period. The remaining known variables not in our study generally fall outside our field of view: V1, V8, V9 (all ab-type RR Lyrae), V6 (an LPV), V15, V16 (both abtype RR Lyrae belonging to the field). V14 is likely not variable, according to the Clement et al. (2001) catalog (see Wehlau \& Butterworth 1990), even though Wehlau \& Sawyer Hogg (1984) provide possible periods around $0.2688 \mathrm{~d}$ for this star.

Most of the variable star candidates listed by Rees \& Cudworth (1991) could not be confirmed in our study. However, based on the position in their finding chart, and as indicated in Table 1, RC133 may be the same star as our NV5, even though the coordinates published in their paper do not exactly match ours. The matching to Rees \& Cudworth was done by triangulation and by eye.

Table 1 summarizes our results. Column 1 gives the star name, whereas Cols. 2 and 3 give their right ascension and declination, respectively (epoch J2000). We derived celestial coordinates for all stars detected in our photometric study by comparison with bright stars obtained from the Two Micron All Sky Survey (2MASS; Skrutskie et al. 2006) catalog stars available through the Infrared Processing and Analysis Center (IPAC) website. Around 1300 stars were available as astrometric references within our image. A third-order polynomial fit, done with the IRAF task mscpeak, produced dispersions of $\sigma \sim 0.2^{\prime \prime}$, consistent with the catalog precision. Column 4 gives our best-fitting 


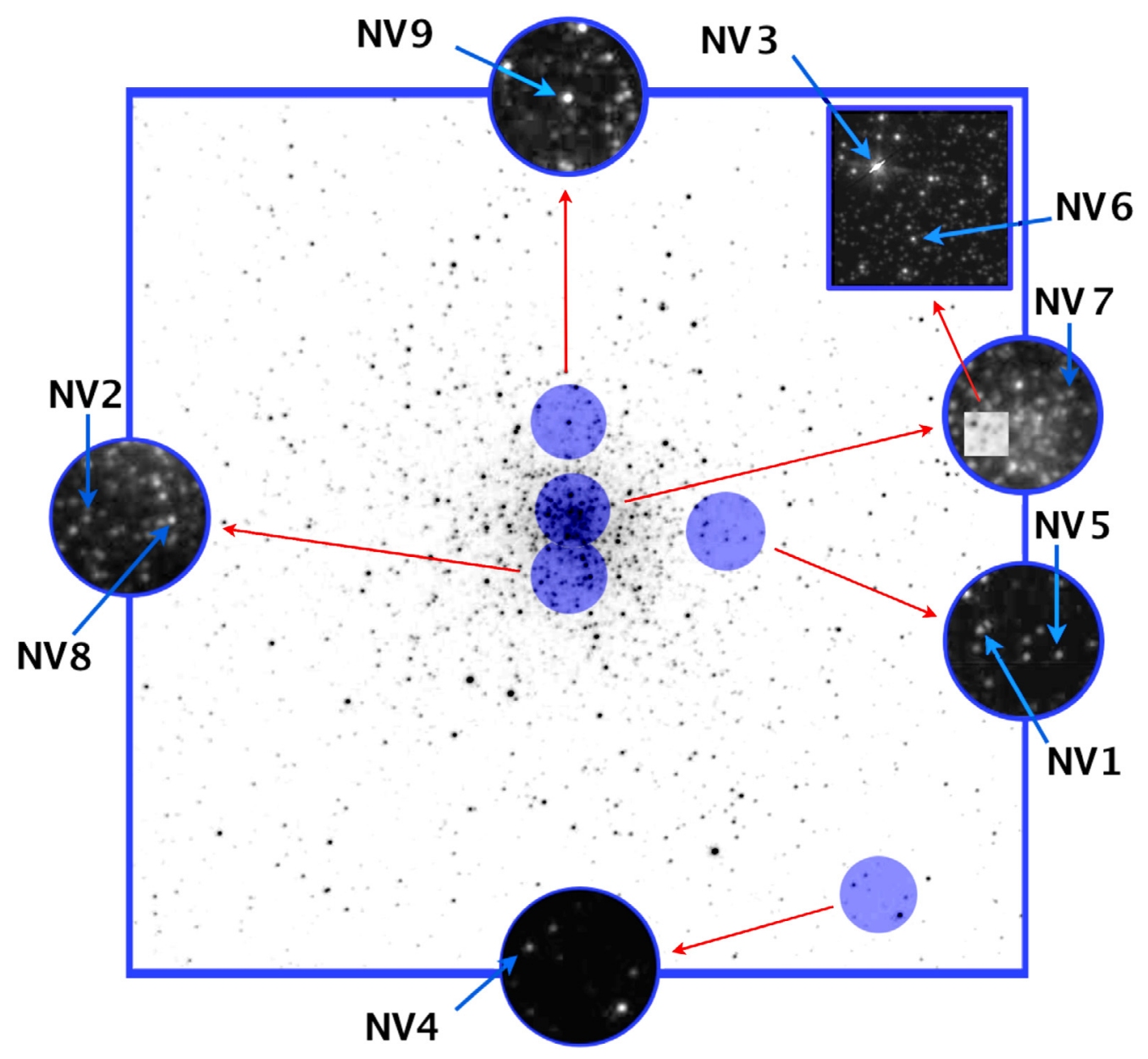

Fig. 1. Finding chart for the newly discovered variable stars in M 28. The large image corresponds to a $V$-band image of the cluster, with the scale of the drawn blue square being $6.5 \times 6.5 \mathrm{arcmin}^{2}$. North is up and East is to the left. The five inner regions of the cluster where new variables have been detected are shown as blue circles superimposed on the main image. Blow-ups are shown for each of these regions, based on an $F 555 W$ ( $V$-band) Hubble Space Telescope image obtained with the WFPC2 camera (ID 6625, PI R. Buonanno). Each such circle covers a region of 30 arcsec diameter. In the top right, a $7.5 \times 7.5 \operatorname{arcsec}^{2}$ subregion of the circle containing NV3, NV6, and NV7 is shown on an expanded scale, for clarity.

period, with as many decimal places as justified on the basis of our data only, whereas Col. 5 gives the previously favored period. Column 6 gives the variability type favored by our data, whereas Col. 7 gives the previously favored variability type. The final column gives an alternative identification for the star, if available. Light curves for the individual variable stars (in relative flux units) are provided in Figs. 2 to 5.

Among the ab-type RR Lyrae in M 28, based on the magnitude values published in the online Clement et al. (2001) catalog, we associate as likely field variables not only stars V15, V16, and V24 (all of which are background stars), but also V9, which is about 1 mag brighter than the other bona fide cluster RR Lyrae stars, and is thus likely a foreground RR Lyrae. The newly discovered RRab, NV1, appears to be a bona-fide cluster member, judging from its position in the Alonso-García et al. (2012) CMDs.

As far as c-type RR Lyrae are concerned, no previous field interlopers had been known. Among the newly detected RRc's, only NV3 appears clearly to be a foreground RR Lyrae, being as it is about 2 mag brighter than other RR Lyrae stars in the cluster, again according to the Alonso-García et al. (2012) CMDs.

For the RR Lyrae stars that are clearly cluster members, one obtains the following average quantities and population ratios: $\left\langle P_{\mathrm{ab}}\right\rangle=0.602 \mathrm{~d}$ (or $0.614 \mathrm{~d}$, if we drop V20); $\left\langle P_{\mathrm{c}}\right\rangle=0.304 \mathrm{~d}$;
$f_{\mathrm{c}}=N_{\mathrm{c}} / N_{\mathrm{c}+\mathrm{ab}}=0.44$ (or 0.47 , if V20 is excluded). In addition, the minimum ab-type RR Lyrae period $\left(P_{\mathrm{ab}, \min }\right)$ and maximum c-type RR Lyrae period $\left(P_{\mathrm{c}, \max }\right)$ are $0.4916 \mathrm{~d}$ and $0.329 \mathrm{~d}$, respectively. These values are quite unusual among known GCs. In particular, both $\left\langle P_{\mathrm{ab}}\right\rangle$ and $P_{\mathrm{ab}, \min }$ are more typical of Oosterhoffintermediate systems (e.g., Catelan 2009; Catelan et al. 2012). As discussed by Catelan et al. (2012), these are the two quantities most strongly defining Oosterhoff status. Still, M 28's $\left\langle P_{c}\right\rangle$ value is clearly more typical of Oosterhoff type I systems, whereas the high c-type number fraction is more common in Oosterhoff type II systems. A "hybrid" Oosterhoff I/II system is accordingly another possibility, which raises the question of whether there might be multiple populations in this fairly massive cluster. Given the known connections between Oosterhoff status and formation history (e.g., Catelan 2009; Clementini 2010; van den Bergh 2011; Catelan et al. 2012), it would certainly be of interest to study the variable star content in M 28 using a more extensive dataset.

To close, we comment on the status of NV8. The star's position in the Alonso-García et al. (2012) CMDs, fairly close to the tip of the red giant branch and at a fairly red color, is consistent with an LPV classification. This is why this star is grouped with other LPVs in Fig. 5. On the other hand, according to our timeseries data, a period around $13.53 \mathrm{~d}$ provides a decent match of 
Table 1. Variable stars in M 28.

\begin{tabular}{|c|c|c|c|c|c|c|c|}
\hline Name & $\begin{array}{c}\text { RA (J2000) } \\
\text { (hh:mm:ss.ss) }\end{array}$ & $\begin{array}{c}\text { Dec } \\
\text { (dd:mm:ss.s) }\end{array}$ & $\begin{array}{l}\text { Period } \\
\text { (days) }\end{array}$ & $\begin{array}{l}\text { Old period } \\
\text { (days) }\end{array}$ & Type & Old type & Other ID \\
\hline$\overline{\mathrm{V} 2}$ & $18: 24: 29.15$ & $-24: 51: 06.6$ & 58 & & LPV & Irr & \\
\hline V3 & $18: 24: 30.17$ & $-24: 50: 18.5$ & 49 & & LPV & Irr & \\
\hline V4 & $18: 24: 30.12$ & $-24: 51: 36.4$ & 13.37 & 13.462 & W Vir & W Vir & \\
\hline V5 & $18: 24: 29.48$ & $-24: 51: 52.6$ & 0.645 & 0.644360 & RRab & RR0 & \\
\hline V7 & $18: 24: 44.19$ & $-24: 50: 30.6$ & & 320.0 & LPV, f & Mira, $\mathrm{f}$ & \\
\hline V10 & $18: 24: 39.51$ & $-24: 53: 26.1$ & & & LPV/Irr & Irr & \\
\hline V11 & $18: 24: 31.48$ & $-24: 51: 33.0$ & 0.542 & 0.542767 & RRab & RR0 & \\
\hline V12 & $18: 24: 43.31$ & $-24: 52: 56.1$ & 0.578 & 0.578228 & RRab & RR0 & \\
\hline V13 & $18: 24: 25.77$ & $-24: 52: 33.6$ & 0.656 & 0.654923 & $\mathrm{RRab}, \mathrm{f} ?$ & RR0 & \\
\hline V17 & $18: 24: 35.84$ & $-24: 53: 15.8$ & 62 & 91.7 & RV Tau & RV Tau & \\
\hline V18 & $18: 24: 36.54$ & $-24: 51: 49.9$ & 0.640 & 0.640151 & RRab & RR0 & \\
\hline V19 & 18:24:30.84 & $-24: 51: 56.0$ & 0.3119 & 0.335 & $\mathrm{RRc}$ & RR1 & \\
\hline V20 & $18: 24: 33.11$ & $-24: 51: 42.9$ & 0.4948 & 0.49774 & RRab? & RR0 & \\
\hline V21 & 18:24:32.94 & $-24: 51: 59.0$ & & 29.93 & LPV & W Vir & \\
\hline V22 & $18: 24: 30.98$ & $-24: 52: 01.8$ & 0.323 & 0.99538 & RRc & BL Her & \\
\hline V23 & $18: 24: 30.25$ & $-24: 52: 03.0$ & 0.292 & 0.29231 & $\mathrm{RRc}$ & RR1 & \\
\hline NV1 & $18: 24: 28.89$ & $-24: 52: 09.9$ & 0.748 & & RRab & & \\
\hline NV2 & $18: 24: 33.59$ & $-24: 52: 32.3$ & 0.297 & & $\mathrm{RRc}$ & & \\
\hline NV3 & $18: 24: 32.31$ & $-24: 52: 32.2$ & 0.323 & & $\mathrm{RRc}, \mathrm{f}$ & & \\
\hline NV4 & $18: 24: 24.13$ & $-24: 54: 36.6$ & 0.261 & & $\mathrm{RRc}$ & & \\
\hline NV5 & $18: 24: 27.67$ & $-24: 52: 15.0$ & 0.311 & & RRc & & RC133? \\
\hline NV6 & $18: 24: 33.45$ & $-24: 52: 10.6$ & 0.308 & & $\mathrm{RRc}$ & & \\
\hline NV7 & $18: 24: 32.17$ & $-24: 52: 00.4$ & 0.329 & & RRc & & \\
\hline NV8 & $18: 24: 33.60$ & $-24: 52: 07.1$ & & 13.53 & W Vir? LPV? & & \\
\hline NV9 & $18: 24: 33.05$ & $-24: 51: 28.9$ & & & LPV & & \\
\hline
\end{tabular}

the light curve (Fig. 5). Such a period is, however, more consistent with a W Virginis (type II Cepheid) classification. Further analysis will clearly be necessary, before the variability nature of this star can be conclusively established.

\section{Summary}

We have presented the results of a new search for variable stars in M 28. Our search has led to the discovery of a number of previously unknown variables, most of which are c-type RR Lyrae stars. The properties of the ab-type RR Lyrae stars are most consistent with an Oosterhoff-intermediate classification, but this is not clearly supported by the properties of the c-type RR Lyrae stars. A "hybrid" Oosterhoff I/II classification is thus possible, raising the question as to whether multiple populations may be present in this fairly massive cluster. More extensive, higherquality datasets will be required to put the properties of this cluster on a firmer basis.

Acknowledgements. We warmly thank C. M. Clement, E. D'Alessandro, J. Kaluzny, E. Tolstoy (the editor), and an anonymous referee for several comments that helped us improve our presentation. Support for M.C. and J.A.-G. is provided by the Ministry for the Economy, Development, and Tourism's Programa Inicativa Científica Milenio through grant P07-021-F, awarded to The Milky Way Millennium Nucleus; by Proyecto Basal PFB-06/ 2007; by Proyecto FONDECYT Regular \#1110326; and by Proyecto Anillo ACT-86. H.A.S. thanks the US National Science Foundation for support under grants AST 0607249 and AST 0707756. This publication makes use of data products from the Two Micron All Sky Survey, which is a joint project of the University of Massachusetts and the Infrared Processing and Analysis Center/California Institute of Technology, funded by the National Aeronautics and Space Administration and the National Science Foundation.

\section{References}

Alard, C. 2000, A\&AS, 144, 363

Alard, C., \& Lupton, R. H. 1998, ApJ, 503, 325

Alcaino, G. 1981, A\&AS, 44, 191

Alonso-García, J., Mateo, M., Sen, B., et al. 2012, AJ, 143, 70

Armandroff, T. E., \& Zinn, R. 1988, AJ, 96, 92

Blazhko, S. 1907, Astron. Nachr., 175, 325

Bramich, D. M. 2008, MNRAS, 386, L77

Catelan, M. 2009, Ap\&SS, 320, 261

Catelan, M., Greco, C., Amigo, P., et al. 2012, AJ, submitted

Clement, C. M., Muzzin, A., Dufton, Q., et al. 2001, AJ, 122, 2587

Clementini, G. 2010, in Variable Stars, the Galactic halo and Galaxy Formation, eds. C. Sterken, N. Samus, \& L. Szabados (Moscow: Sternberg Astronomical Institute), 107

Contreras, R., Catelan, M., Smith, H. A., Pritzl, B. J., \& Borissova, J. 2005, ApJ, 623, L117

Contreras, R., Catelan, M., Smith, H. A., et al. 2010, AJ, 140, 1766

Cudworth, K. M., \& Hanson, R. B. 1993, AJ, 105, 168

Davidge, T. J., Côte, P., \& Harris, W. E. 1996, ApJ, 468, 641

Harris, W. E. 1996, AJ, 112, 1487

Kuehn, C. A., Smith, H. A., Catelan, M., et al. 2011, AJ, 142, 107

Lyne, A. G., Brinklow, A., Middleditch, J., et al. 1987, Nature, 328, 399

Oosterhoff, P. Th. 1939, Observatory, 62, 104

Oosterhoff, P. Th. 1944, Bull. Astron. Inst. Neth., 10, 55

Rees, R. F., \& Cudworth, K. M. 1991, AJ, 102, 152

Rosenberg, A., Piotto, G., Saviane, I., \& Aparicio, A. 2000, A\&AS, 144, 5

Sawyer, H. B. 1949, AJ, 54, 193

Skrutskie, M. F., Cutri, R. M., Stiening, R., et al. 2006, AJ, 131, 1163

Stellingwerf, R. F. 1978, ApJ, 224, 953

Testa, V., Corsi, C. E., Andreuzzi, G., et al. 2001, AJ, 121, 916

van den Bergh, S. 2011, PASP, 123, 1044

Wehlau, A., \& Butterworth, S. 1990, AJ, 100, 686

Wehlau, A., \& Clement, C. M. 1990, IBVS, 3450, 1

Wehlau, A., \& Sawyer Hogg, H. 1982, IBVS, 2171, 1

Wehlau, A., \& Sawyer Hogg, H. 1984, AJ, 89, 1005 
G. Prieto et al.: Variable stars in M $28(R N)$
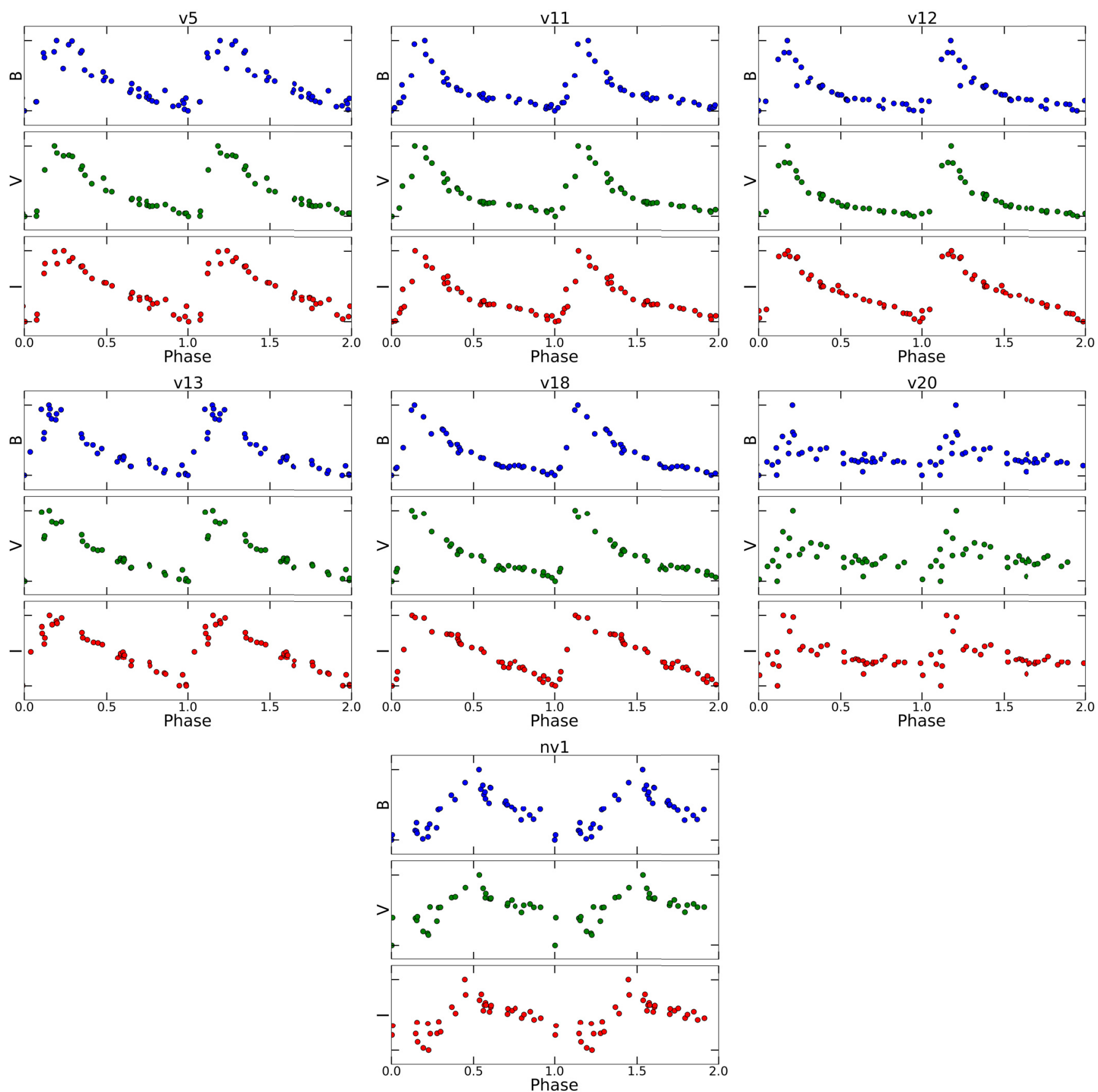

Fig. 2. Light curves of the ab-type RR Lyrae detected in this work, in the $B$ (top panels), $V$ (middle panels), and $I$ (bottom panels), respectively. 

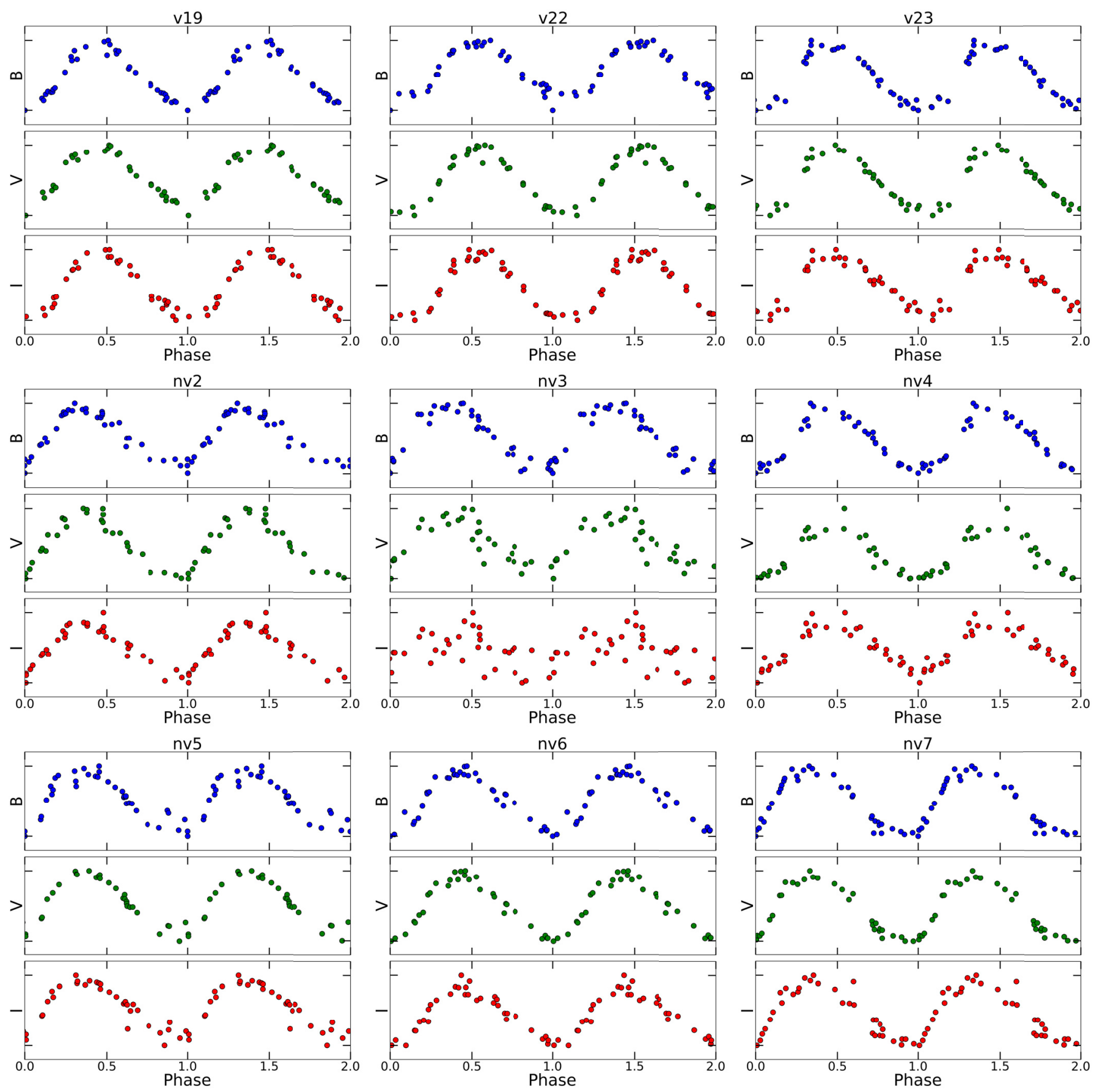

Fig. 3. As in Fig. 2, but for the c-type RR Lyrae. 
G. Prieto et al.: Variable stars in M $28(R N)$
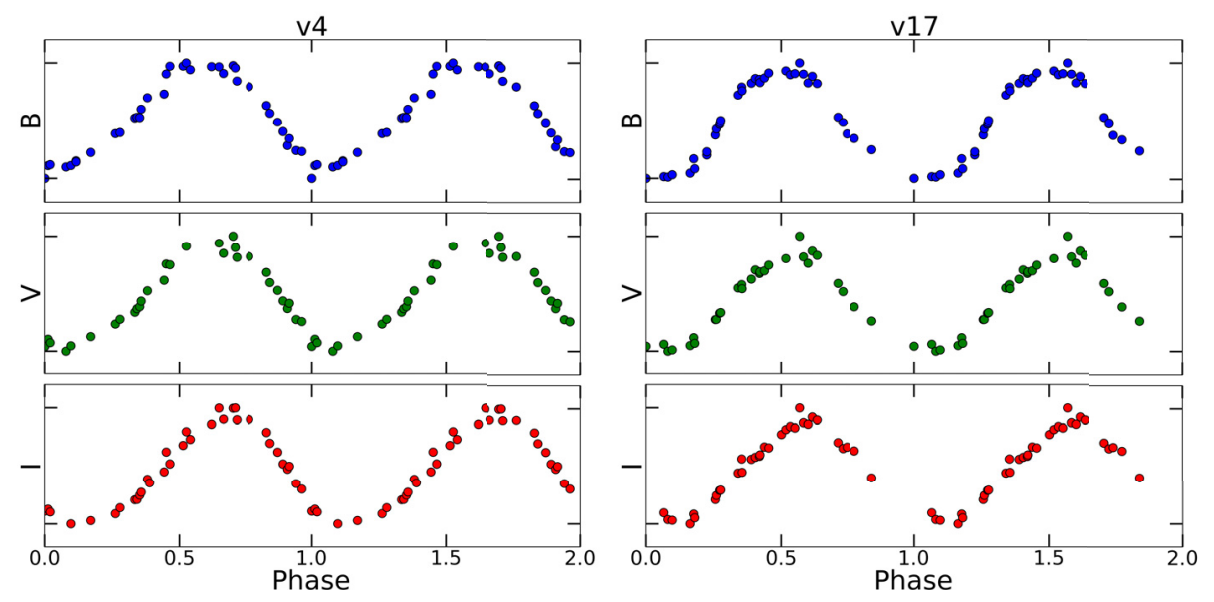

Fig. 4. As in Fig. 2, but for the type II Cepheids. 

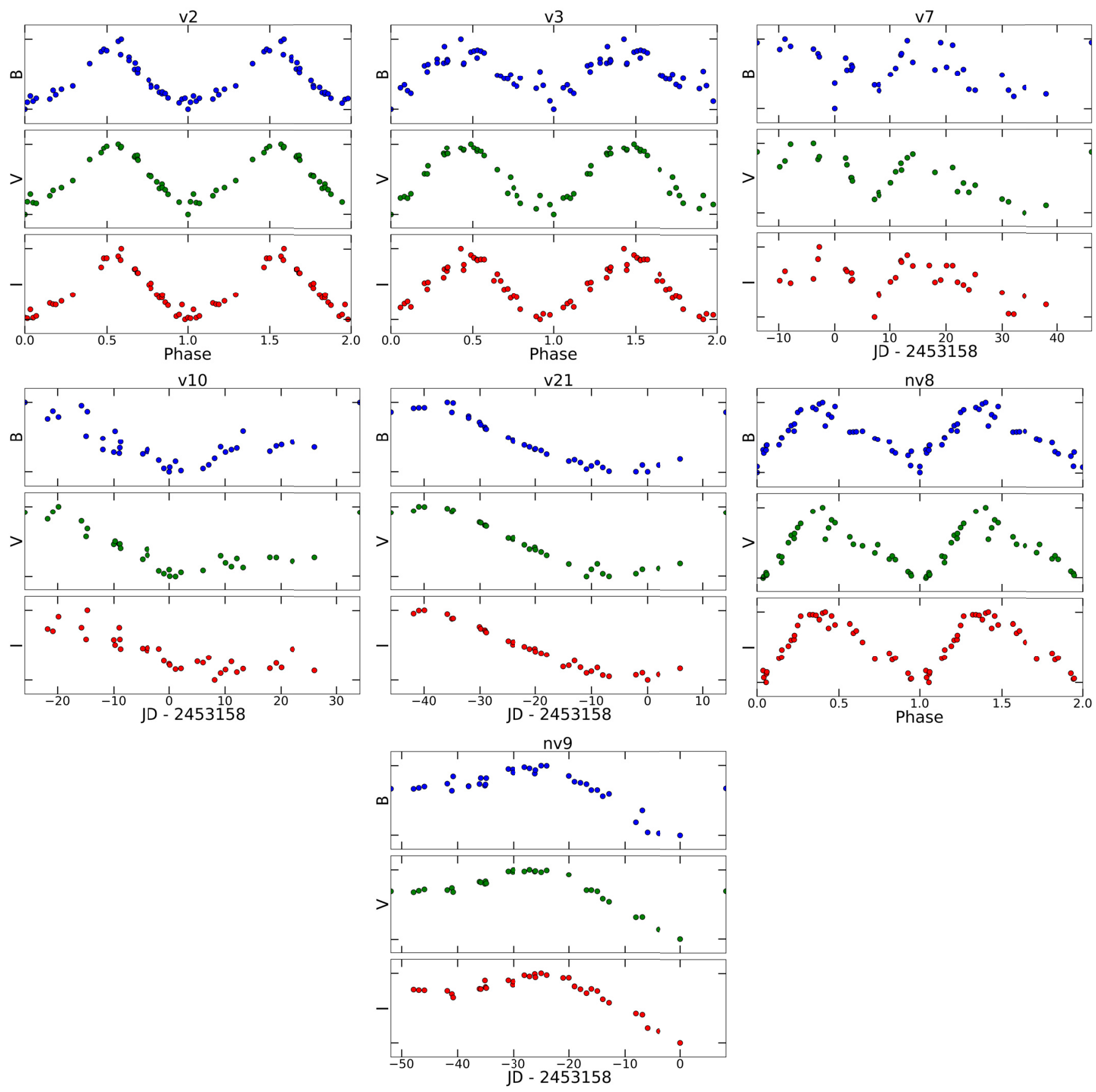

Fig. 5. As in Fig. 2, but for the LPVs. 